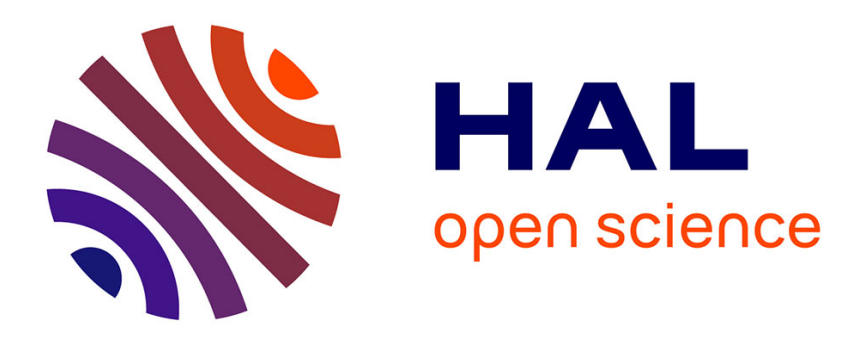

\title{
Paul à Québec, le génie des lieux comme patrimoine identitaire
}

\author{
Sylvie Dardaillon, Christophe Meunier Meunier
}

\section{To cite this version:}

Sylvie Dardaillon, Christophe Meunier Meunier. Paul à Québec, le génie des lieux comme patrimoine identitaire. 2013. halshs-01181069

\section{HAL Id: halshs-01181069 \\ https://shs.hal.science/halshs-01181069}

Preprint submitted on 29 Jul 2015

HAL is a multi-disciplinary open access archive for the deposit and dissemination of scientific research documents, whether they are published or not. The documents may come from teaching and research institutions in France or abroad, or from public or private research centers.
L'archive ouverte pluridisciplinaire HAL, est destinée au dépôt et à la diffusion de documents scientifiques de niveau recherche, publiés ou non, émanant des établissements d'enseignement et de recherche français ou étrangers, des laboratoires publics ou privés.

\section{(c) (1) $\$$}

Distributed under a Creative Commons Attribution - NonCommercial| 4.0 International 


\section{Paul à Québec, le génie des lieux comme patrimoine identitaire}

Sylvie Dardaillon, Docteure en littérature, professeure de Lettres à l'ESPE Centre Val de Loire (Université d'Orléans) Équipe Dynadiv (Université de Tours) Christophe Meunier, Doctorant en géographie, professeur d'HistoireGéographie à l'ESPE Centre Val de Loire (Université d'Orléans)

UMR 5600 Environnement, Ville et Société, ENS de Lyon

Depuis bientôt quinze ans, le dessinateur québécois Michel Rabagliati fait de sa propre vie le terreau des aventures de Paul, son alter-ego. Paul est un «héros ordinaire». Sa vie est celle de tout-un-chacun, avec ses joies, ses peines. En 2009, l'auteur remportait le prix du Jury du Festival d'Angoulême avec son album Paul à Québec. Graphic novel de 187 pages, la bande dessinée est centrée sur le beau-père de Paul, Roland Beaulieu. Ce dernier, atteint d'un cancer généralisé, vit une véritable descente aux enfers. Rien n'est épargné aux lecteurs depuis les premiers symptômes jusqu'à la phase terminale. C'est l'occasion pour Rabagliati de rendre hommage à son propre beau-père qu'il avait en admiration. Roland est un Québécois " pure souche », né près des remparts de la "Vieille capitale», un self-made man exemplaire. C'est cet album que nous avons choisi de privilégier, afin d'analyser comment Rabagliati parle, à sa façon, de l'identité québécoise.

En 2009, Michel Labrie, dans Le Mouton Noir, journal en ligne québécois, voyait en Paul le témoin d'une « identité québécoise » qu'il définissait de la manière suivante :

Paul témoigne de l'identité québécoise. Il la décline par une juste et fine mise en scène des valeurs qui sont les exigences mêmes de notre dignité : l'amour et l'amitié, la famille et le travail.. ${ }^{1}$

Si amour, amitié, famille et travail participent d'une construction identitaire, l'œuvre de Rabagliati recèle de notre point de vue d'autres composantes de ce que serait «l'identité québécoise ». Doit-on la chercher dans la langue ? dans les relations sociales ? dans l'habiter ?... C'est ce que nous nous proposons de faire dans la suite de cet article en interrogeant d'abord les représentations d'une spatialité canadienne ou québécoise véhiculées par l'album; puis les usages linguistiques et culturels et enfin la construction d'une identité à partir des lieux mis en scène dans l'œuvre de Rabagliati.

\section{Une spatialité canadienne?}

La pratique des espaces par les personnages de Paul à Québec est déterminante de l'identité construite par ces mêmes personnages. La délimitation de leur espace vécu, la relation qu'ils tissent avec certains lieux de leur histoire personnelle, leur manière de parcourir un territoire qu'ils ont fait leur participent, voire constituent presque globalement, leur identité propre.

Paul à Québec, à l'instar de Martine à la ferme ou Caroline à la mer, est un album-découverte. Comme les deux héroïnes, Paul part à la rencontre d'une ville, de gens qui lui sont proches et qui l'habitent. A contrario des autres albums de la série, il y est assez souvent observateur et les dernières scènes sont en cela très révélatrices : Paul (alias Michel Rabagliati) raconte en témoin les dernières heures de son beau-père entouré de sa femme et de ses trois filles.

\subsection{De Montréal à Québec}

Michel Rabagliati a décidé de donner à cet album, très intime, centré sur la vie et les derniers mois du père de sa femme, le titre de Paul à Québec alors que l'essentiel de l'action du graphic-novel se passe dans la banlieue de Montréal. Québec n'est évoquée qu'en trois occasions: au début de l'album avec la réunion familiale à Saint-Nicolas, dans la partie centrale consacrée au récit en analepse de la vie de Roland Beaulieu et à la fin, avec l'enterrement de ce dernier. Il semblerait donc que la ville de Québec revête pour Paul une dimension symbolique. Il s'agit de la « vieille capitale », le berceau de la Nouvelle France, la ville des origines.

\footnotetext{
${ }^{1}$ Michel Labrie, « Paul, le héros de la vie ordinaire » dans Le Mouton Noir, $1{ }^{\text {er }}$ juillet 2009 http://www.moutonnoir.com/2009/07/paul-le-heros-de-la-vie-ordinaire/
} 
L'évocation de Québec se fait ainsi à partir de deux lieux de l'agglomération : sa banlieue, Saint-Nicolas et son quartier historique, le «Vieux Québec». Dans les deux cas, il s'agit d'un retour aux sources : aux sources de l'enfance de Lucie, aux sources des premiers occupants du Québec.

Saint-Nicolas, aujourd'hui un des quartiers de la ville de Lévis, dans la banlieue sud de Québec, sur la rive droite du Saint-Laurent, est une des plus vieilles paroisses du Québec. C'est là que Roland Beaulieu a décidé de passer sa retraite au début de l'album, c'est là que Lucie retrouve ses oncles et tantes. C'est également là que Lucie et ses sœurs passaient leurs vacances lorsqu'elles étaient enfants. La famille Beaulieu, qui vivait alors à Orsainville, dans les quartiers résidentiels du nord de Québec, louait tous les étés un petit chalet au bord de la rue de la Montagne, au pied des contreforts appalachiens. L'attachement à cet endroit est tout à fait perceptible aux pages 28-29, quand Paul, sa femme Lucie et ses belles sœurs et beaux-frères se baladent dans les environs du nouveau chalet acheté par Roland. Deux vues zénithales mises côte à côte montrent les transformations de ce paysage qui, dans les années 60, était encore un endroit quasi-sauvage, une terre de pionniers, à l'écart, semble-t-il, de la "Révolution tranquille» des années 60-70. L'urban sprawl mis en évidence dans la vignette en regard et la modernisation de la route Marie-Victorin qui longe le Saint-Laurent ont eu raison de cette petite paroisse "champêtre » aujourd'hui périurbaine.

\section{Document 1}

Déplacements de Roland Beaulieu dans Québec

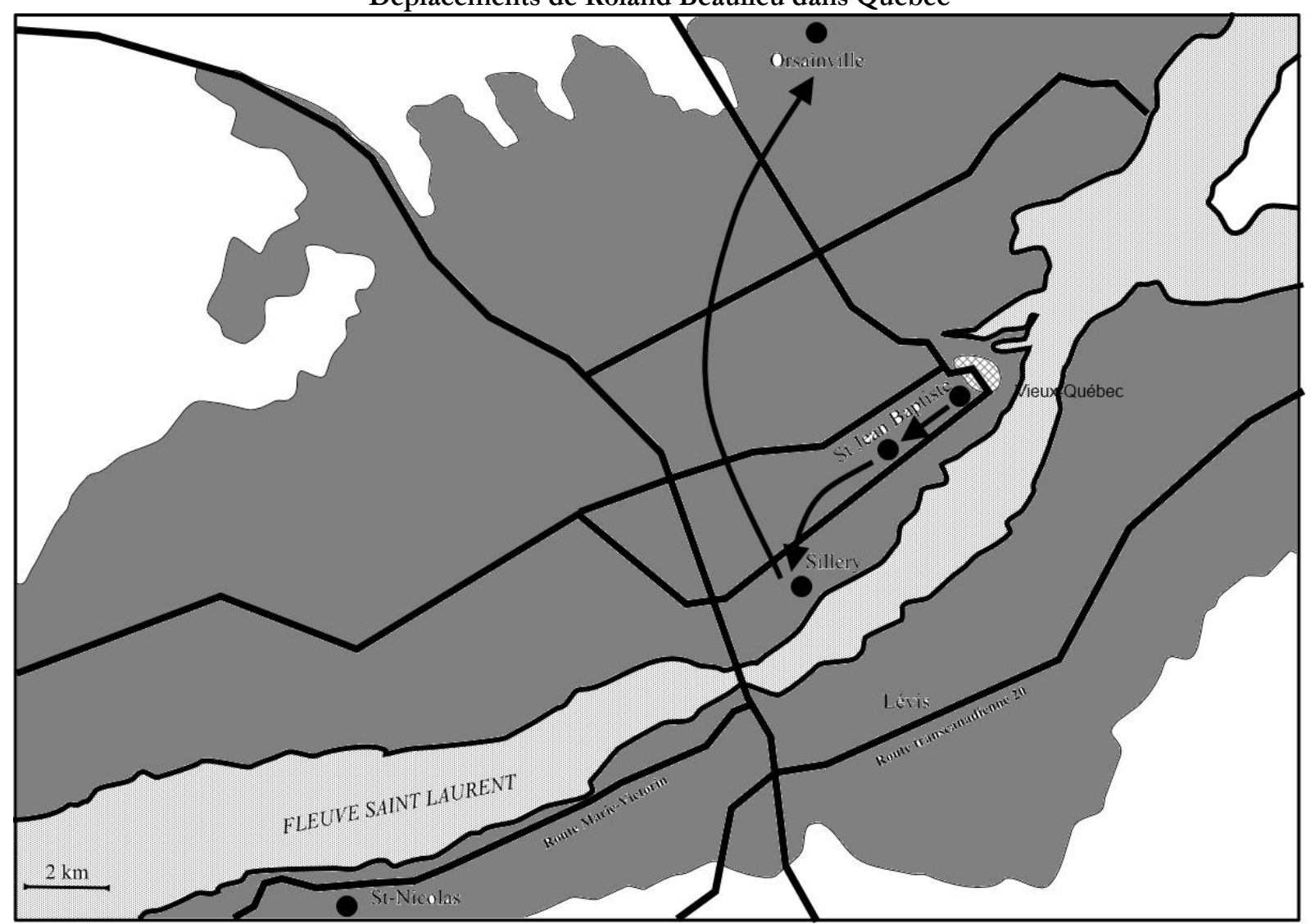

C'est d'ailleurs près de l'église Saint-Nicolas, dans un cimetière situé au bord du SaintLaurent, que Roland Beaulieu a décidé d'être enterré. C'est ce décor que Rabagliati a choisi de livrer aux lecteurs sur la première de couverture. Paul, mains dans les poches, l'écharpe au vent, debout sur la terrasse du chevet de l'église, regarde pensif vers la droite (en l'occurrence l'ouest), vers Québec. Derrière lui, un bateau descend le Saint-Laurent. Aucun cliché «touristique » de Québec n'est offert pour combler l'horizon d'attente du lecteur appâté par le titre. Le projet de 
l'auteur est annoncé : c'est du Québec de Paul d'abord dont il sera question et peut-être de celui dans lequel certains lecteurs initiés se reconnaittront, l'album créant ainsi une complicité identitaire avec son lectorat.

Aux pages 60 à 62, Paul évoque l'enfance de son beau-père dans le milieu des années 1930. Le lecteur est alors transporté dans une autre partie de Québec, celui de la rue de la Tourelle et de l'hospice Saint-Joseph de la Délivrance dans le quartier Saint-Jean-Baptiste, c'est-à-dire dans les anciens faubourgs de Vieux-Québec, la ville fondée par Champlain en 1608. L'itinéraire de Roland Beaulieu fait de lui un enfant pauvre du Québec traditionnel, du «vrai» Québec. Né dans les franges de la seule cité fortifiée nord-américaine, Roland a grandi abandonné de ses parents et s'est construit lentement, gravissant les échelons de l'échelle sociale. Paul est en admiration devant ce beau-père qui s'est fait lui-même. Il constitue le chêne d'une famille soudée : il en est les racines les plus profondes prises dans le sol des premiers pionniers, le tronc le plus solide dont l'écorce s'est épaissie au fil des épreuves de la vie et le ramage le plus dense.

\section{Document 2}

\section{Couverture de Paul à Québec (2009)}

\subsection{Roland Beaulieu ou le mythe du pionnier}

Pour Paul, Roland Beaulieu s'est construit à l'image des pionniers qui ont fait de la Nouvelle France le Québec. Roland Beaulieu est un self-made man parti de rien qui fut toute sa vie un grand voyageur, trouvant dans la mobilité son moteur vital, à la recherche sans doute du lieu idéal, du « beau » lieu pour y planter définitivement sa tente sans jamais y parvenir réellement.

C'est dans cette extrême mobilité qu'il faut trouver une spatialité toute canadienne ou nordaméricaine. Comme le montre le document 1, la vie de Roland Beaulieu est un parcours à travers le Québec, parcours centrifuge jusqu'à la maturité et centripète jusqu'à sa fin. Parti de la "ville capitale », il explore jusqu'aux confins du Québec. La ville qui l'a vu naître et la province qu'il parcourt pendant sa jeunesse deviennent son espace de vie, son territoire qu'il s'approprie au fil d'une pratique itinérante.

\section{Document 3}

Déplacements de Roland Beaulieu autour de Québec 


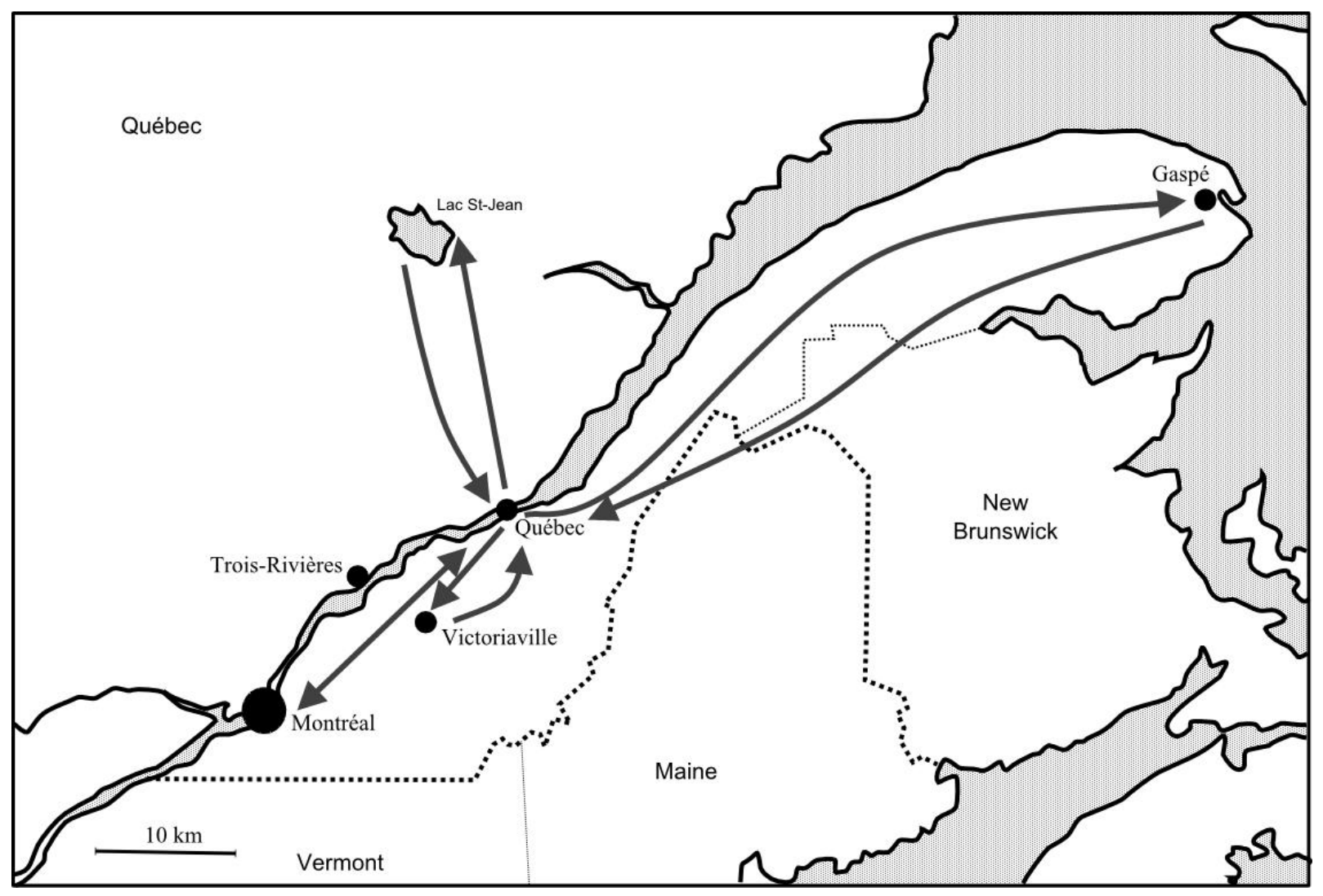

À la page 60, Paul retrace l'enfance malheureuse de Roland dans le faubourg de Sain-JeanBaptiste. Très tôt mis à la porte de chez lui, placé à l'hospice Saint-Joseph de la Délivrance puis chez les pères maristes à Sillery, il va arpenter à pied tout le Vieux-Québec et les faubourgs, vivant de maigres expédients et de menus larcins. Sa période d'errance dans le centre de Québec est une longue phase de territorialisation. C'est au cœur du quartier Haute-ville, au coin de la rue Aberdeen qu'il finit par rencontrer M.Thioret, un commerçant qui va lui donner sa chance. Au contact de M. Thioret, Roland se rachète une conduite et trouve un père de substitution. C'est pour lui comme une seconde naissance qui le conduit à explorer encore plus loin.

À 19 ans, il quitte la rue Aberdeen et Québec pour le Bout du lac Saint-Jean. Il va travailler là comme bûcheron, à $200 \mathrm{~km}$ au nord de la «ville capitale ». Cette fuite vers des terres inhospitalières constitue une sorte d'épreuve pour le jeune garçon qu'il était alors. Il s'endurcit, se bonifie. Lorsqu'il revient chez M.Thioret, c'est un homme qui se présente à son mentor. Roland a l'idée de fonder une famille. Il rencontre Lisette et, pour améliorer le quotidien du jeune ménage, Roland devient commis voyageur. Son travail le conduit tous les jours de l'année sur les routes. Peu à peu, Roland tisse la trame de son territoire toujours plus grand, s'étirant le long du SaintLaurent, entre Victoria-ville et Gaspé.

Cette phase d'ultramobilité, centrifuge, prend fin dès lors que son épouse attend leur premier enfant. Le couple s'installe à Orsainville dans une banlieue pavillonnaire au nord de Québec; puis à Boucherville, dans la banlieue nord de Montréal. Cette dernière étape correspond à la fin de l'ascension sociale du petit commis voyageur devenu responsable commercial d'une grande entreprise, C.N.A. Distribution. Car à cette ultramobilité assagie correspond une amélioration du niveau de vie et de l'habitat pour la famille Beaulieu. Le dernier déménagement à Saint-Nicolas restera anecdotique dans la mesure où, au bout d'un an à peine, Roland et Lisette remménagent à Montréal, dans un condominium, «à proximité d'un IGA ${ }^{2}$ » (cf. p.59 b.3 c.1).

Les déplacements géographiques de Roland se terminent à La Chênaie, la maison de soin située dans la périphérie de Montréal (à Beloeil...). À partir de là, la vie de Roland s'arrête

\footnotetext{
${ }^{2}$ Chaine de supermarché québécois : Independent Grocers Alliance
} 
lentement comme si la mobilité spatiale était son oxygène. Sa vie sur terre prend fin dans son lit d'hôpital au milieu des chênes mais c'est à Saint-Nicolas qu'il reprend une autre forme de vie, non plus terrestre celle-là mais spirituelle. Aux dernières pages de l'album, son âme s'échappe de sa tombe pour saluer une dernière fois sa petite-fille Rose, avant de s'évader pour un dernier voyage dans les cieux. L'effet de zoom inversé des dernières cases participe du dernier mouvement et le lecteur accompagne en quelque sorte les derniers «pas » de Roland vers l'au-delà.

Ce que retranscrit Rabagliati dans les onze pages qui évoquent la vie de son beau-père c'est la genèse spatiale du Québec de Roland Beaulieu. Le récit de sa vie est le récit de ses mouvements, de ses parcours à travers une partie du Québec. C'est également le récit d'espaces. Cette mobilité caractéristique des sociétés nord-américaines participe de l'identité même de Roland Beaulieu. Il existe grâce à elle, se définit en tant que Québécois avec elle. Ce sont les lieux qu'ils traversent (la rue la Tourelle, le coin de la rue Aberdeen, le Bout-du-lac-Saint-Jean), qui jalonnent son espace vécu. Affectés d'une histoire, ils ponctuent son récit de vie autant que son propre récit spatial.

\subsection{Une territorialisation par les lieux}

Les premières pages de l'album témoignent particulièrement bien de cet attachement aux lieux constitutifs de nos vies. C'est par les lieux que Paul évoque son attachement à son beau-père et à sa belle-famille. À ce titre, les deux planches du début consacrées à l'arrêt de Paul, Lucie et Rose au restaurant «Le Madrid» sont particulièrement intéressantes. Géographiquement, le restaurant se trouve à la sortie 202 de l'autoroute transcanadienne 20, près de Drummondville, à mi-chemin entre Montréal et Québec. Paul y est frappé par le mélange des genres «monster trucks et faux dinosaures ». Mélange des genres il y a à coup sûr car le restaurant est également situé à mi-chemin également entre les provinces anglophones de l'Ontario et des Maritimes. Le service de l'établissement y est bilingue. D’une autre manière, si Montréal est la ville québécoise multiculturelle, Québec s'avère être la ville québécoise par excellence, la « capitale culturelle ».

C'est ce qui transparaît quelque peu à travers les pages 25 à 29. Lucie, Paul, Monique, Suzanne et le compagnon de celle-ci se baladent dans Saint-Nicolas à la recherche de leur enfance. L'itinéraire qu'ils empruntent est encore un récit d'espace autant qu'un récit de vie et, en remontant la rue des Pionniers, ils remontent le temps.

Les repères spatiaux sont extrêmement précis et ont été sélectionnés avec soin par Rabagliati. L'église Saint-Nicolas (p.25) qui date de 1962 tranche avec les maisons victoriennes du village. Son style architectural particulier, dû à André Gilbert, représente le carénage d'un bateau, rappelant le passé de marins des habitants de la localité. Un peu plus loin sur la même rue des Pionniers, le Centre de diffusion d'art et du patrimoine (p.26 b3 c1) pavoise aux couleurs du Québec. Le groupe passe devant le funérarium Claude-Marcoux qui sert de décor à la Maison Requiem (p.173 b.1 c.1 à 6) pour arriver devant le garage de Gosselin, repère spatial de l'endroit où se trouvait le chalet des vacances de l'enfance.

Cette remontée dans le temps est également une reterritorialisation d'un espace qui a fait partie de la vie des trois sœurs. La déambulation ranime les souvenirs et le décor se construit peu à peu au fil des cases. Entre tradition et modernité, Saint-Nicolas devient le village-idéal d'une culture québécoise qui s'est développée au gré du Saint-Laurent, des arrivées successives de pionniers français, d'une occupation britannique et d'une influence américaine.

\section{Paul à Québec : la mise en scène d'une identité québécoise ou canadienne ?}

Si nous observons à présent cette culture québécoise, voire cette identité, qui constitue le terreau de l'œuvre de Rabagliati et en particulier celui de Paul à Québec, nous observons alors qu'elle se base à la fois sur une conscience politique, sur un usage vivant d'une langue minoritaire sur le territoire nord-américain, le français, et sur des pratiques, des us et coutumes, plus ou moins partageables par les lecteurs européens. De cette culture, Rabagliati contribue à construire une représentation complexe. Il donne en effet une image plurielle de la province de Québec, présentée à la fois comme une unité et comme un tissu de particularismes régionaux : Lucie 
explique que « finir ses croûtes de pizza avec du beurre, y a juste à Québec que ça existe » (p..10). Dans une note de bas de planche, Rabagliati met également l'accent (non sans humour) sur les variations phonologiques entre Montréal et Québec : « une autre différence fondamentale entre les habitants de Québec et ceux de Montréal, la prononciation des trois mots [...] arrête, poteau et baleine » (p..36).

\subsection{Québec, lieu de la cristallisation indépendantiste ?}

«Vive le Québec libre!» crie Lucie, la femme de Paul, à son arrivée chez ses parents (Pl.13), au vieux Saint-Nicolas. Le slogan fonctionne à la fois comme une formule phatique (annoncer son arrivée, nouer le contact), comme un signe de reconnaissance identitaire (appartenance à une tribu) et comme un private Joke récurrent qui déclenche une réaction vive de sa jeune sœur Monique : «Québec stuuuu!»(p..13). Sur les deux planches suivantes, les jeunes couples trinquent à l'indépendance du Québec, Paul évoque les feux de camp familiaux animés par les chansons de Charlebois...

Mais cette identité québécoise affirmée n'est pas sans poser problème. Rabagliati montre en effet les "tensions» que peut occasionner la revendication indépendantiste au sein des familles, l'absence d'unanimité. "Ah! Commencez pas avec ça vous autres les séparatisses!», proteste Roland Beaulieu (p.14) qui explicite clairement sa position à la vignette suivante : "Not' pays c'est le Canada! Pis not' province c'est le Québec! C'est de même que c'est, pis c'est de même que ça doit rester! ». Le soulignement, inscrit dans la bulle par l'auteur, marque ici le phrasé du personnage qui martèle son discours, scande son credo. De plus, la symétrie syntaxique de sa dernière phrase, doublée d'un usage gnomique du présent, de la valeur essentielle du verbe, du glissement d'être à devoir, font de cette affirmation un acte de parole au sens plein.

Par ailleurs, lorsque au cours de leur promenade dans le vieux Saint-Nicolas, les jeunes couples croisent une voiture d'indépendantistes, ils en viennent eux-mêmes à douter de l'avenir d'un Québec libre, avec le sentiment nostalgique d'avoir « raté le bateau » (p.25). Pour eux «la soupe a viré frette en sibole» (p.26). Monique considère même que les hésitations des Québécois les ont décrédibilisés aux yeux du monde entier qui "devait se dire: "Coudonc, ils vont-tu se brancher pis la faire leur hostie d'indépendance, qu'on passe à aut'chose ?” ».

\section{Document 4}

Reproduction de la page 26

L'intégralité de la page 26, dont les allusions ne sont pas toutes immédiatement perceptibles pour des non-québécois, se fait alors l'écho d'une analyse à la fois désabusée et teintée d'humour de ce rendez-vous raté avec l'histoire. À l'évocation du référendum de 1995, Paul se livre à une imitation irrésistible de René Lévesque, surnommé Ti-Poil par ses concitoyens, plus populaire à ses yeux que Jacques Pariseau, premier ministre de l'époque, surnommé quant à lui Monsieur.

\subsection{Le français comme langue identitaire?}

Cette posture un rien nostalgique mais en rien revendicative reflète peut-être celle de l'auteur: modérée, pragmatique... Il en est de même au plan linguistique. Faisant œuvre francophone, Rabagliati propose dans ses albums une pratique linguistique ne diffère en rien de l'usage commun au Québec en ce qu'elle marque une nette différenciation entre oral et écrit. Cartouches et bulles rendent assurément compte d'un double usage du français entre une langue scolaire, standard et les variations de la langue parlée. Tandis que les cartouches narratifs ou explicatifs sont écrits dans un français international, les bulles nous donnent à entendre un français québécois populaire, spécifique, voire du joual, sociolecte issu de la culture populaire urbaine de la région de Montréal, légitimé dans les années soixante au sein de la revue politique et culturelle Parti pris et introduite dans l'écriture littéraire par des auteurs comme Michel Tremblay. 
Cet usage du joual renvoie donc à des débats de société qui ont eu lieu et à des questions qui se sont posées (et se posent encore aujourd'hui) dans la controverse linguistique.

La langue de Rabagliati est marquée par des spécificités phonétiques parfois issues du moyen français : «y » pour «il, elle »; « moué » pour « moi »; « icitte »pour « ici »; « ouain » pour « oui »... Le français qu'il nous donne à entendre est également émaillé de spécificités lexicales : «L'eau doit être frette ${ }^{3}$ » (p.15) ; ses gendres se revendiquent indépendantistes pour "étriver», c'est à dire «taquiner» Roland Beaulieu (p.14); «La p'tite s'est enfargée ${ }^{4}$ dans la lampe » et réveille toute la famille (p.18); les somnifères distribués par Monique à ses sœurs et beaux-frères les ont «fessés» c'est-à-dire assommés (p.21); maintenant qu'elles sont ados, les filles de Monique «foirent au lit jusqu'à 11 heures...» (p.21)... Ces particularités de la langue courante témoignent d'un ancrage historique du français québécois, tout comme l'usage des "sacres », ces jurons qui manifestent un certain détachement par rapport aux choses religieuses et plus particulièrement à la religion catholique très (trop) présente dans tous les aspects de la vie québécoise. Sacrer permettait de se libérer symboliquement du joug imposé par l'église. Rabagliati nous donne à entendre nombre de sacres et leurs variations: "tabaslac » pour "tabarnac», " batêche » pour «baptême », "câline » pour «calice »... De plus, la variation lexicale se double de variations syntaxiques puisque le sacre peut-être à la fois en position d'adverbe, d'interjection mais aussi d'adjectif : «C'est quoi c'te simonac de machine-là ? » (p.19).

Toutefois, l'usage du français n'apparaît pas vraiment comme la revendication d'une langue identitaire, de notre point de vue. Les héros de Rabagliatti, tout comme la plupart des québécois (et nombres de francophones d'ailleurs), pratiquent en effet un français hybride, métissé d'anglais. Le passage d'une langue à l'autre se fait tout naturellement dans le flux de la parole: «Viens, Rose, ton grilled-cheese est servi» (p.10) annonce Lucie à sa fille; "Shit ! Pourquoi j'ai jamais mon Kodak quand il me le faut» se plaint-elle à la page suivante ; "Tire la plogue, on le rebranchera demain », conseille Paul à son beau-frère (p.19); "Simonac, Monique! T'es une vraie pusher!», s'exclame le même Paul à la page suivante; Suzanne, la sœur aînée de Lucie déclare fumer« juste pour le fun»; le raton capturé par grand-'pa est «toute cute» (p.30); «ben là, laissez-moi deux secondes pour checker mes affaires», demande Paul qui «stagne » en jouant à J'achète...

\subsection{Une identité complexe}

Au-delà de la tentation indépendantiste ou du particularisme linguistique, l'univers narratif de Rabagliati, s'ancre profondément dans une culture québécoise presque stéréotypique. La génération de Roland Beaulieu choisit de se retirer dans les grands espaces, à la campagne, d'opérer un retour aux sources. On peut en prendre pour exemple Gilles et Fernande, l'oncle et la tante de Lucie (pages 34 à 36). Ils se sont offert pour la retraite « une magnifique terre de près d'un kilomètre de long ». Ce territoire leur permet de réinvestir des valeurs culturelles communes. La première peut-être, être en mesure de produire son propre sirop avec l'eau des érables de sa terre, dans la traditionnelle «cabane à sucre» et pouvoir offrir les emblématiques «suçons au sirop d'érable » à ses petits-enfants. La vaste terre de Gilles et Fernande devient également le théâtre d'une activité productive (remise en état, sculpture, cuisine...) qui fait écho à l'hommage fait par ses collègues au self-made-man qu'est Roland Beaulieu. Enfin, cette terre permet à la famille de se forger une identité forte, "des souvenirs inoubliables » basés sur des pratiques sportives de plein air: randonnées à cheval, chasses au trésor, glissades dans la neige... Ces références culturelles typiquement québécoises sont néanmoins plus largement partagées avec tous les canadiens. Le goût pour les grands espaces, la faculté à subvenir soi-même à ses besoins ainsi que le «culte » voué à la famille nous semblent d'ailleurs dépasser le cadre canadien. Se dessine alors, au-delà de l'identité canadienne, une identité fondamentalement nord-américaine au fil des pages

\footnotetext{
${ }^{3}$ Variation de « froid » issue de la langue d'oül

${ }^{4}$ Du vieux français enfergier : entraver en plaçant des chaînes ou des fers, généralement à un animal.
} 
de l'album Paul à Québec, placé sous l'égide de Léonard Cohen par le biais d'une citation liminaire : "You live your life as if it's real, a thousand kisses deep ».

\section{Les lieux comme inducteurs d'un récit identitaire}

Ce que fait Rabagliati à travers l'évocation des lieux dans Paul à Québec participe d'un processus récurrent dans toute la série des Paul. On pourrait y voir une sorte d'idiosyncrasie chez l'auteur. Les albums, pris successivement, sont autant de récits d'espaces qui raniment les décors de l'enfance de Paul/Michel. Comme pour Roland Beaulieu, ces récits d'espaces donnent vie au personnage de Paul et lui confèrent son identité propre. Dans une interview accordée à Xavier Guilbert en 2011 pendant le Festival d'Angoulême, Michel Rabagliati disait :

C'est cette envie de témoigner d'un pays. C'est une préoccupation majeure dans mon œuvre. Les personnages évoluent sur un décor, mais évoluent dans une société, évoluent dans l'air du temps. Je me sens comme une espèce de mission de parler d'un Québec contemporain. ${ }^{5}$

\subsection{Paul est montréalais}

Montréal est un personnage important dans la majeure partie des albums et Rabagliati aime à montrer un grand nombre de lieux que le lecteur assidu peut s'amuser à identifier ${ }^{6}$. Dans Paul à la campagne (1999), c'est le quartier Rosemont qui nous est dépeint à travers quelques endroits particuliers comme l'aire de jeu entre la $20^{\mathrm{e}}$ et la $21^{\mathrm{e}}$ Avenue; dans Paul en appartement (2004) il s'agit du Plateau-Mont-Royal que Paul et Lucie habitent.

Le nord de l'île de Montréal est l'espace circonscrit à la vie de Paul depuis sa naissance (20 e Avenue) jusqu'à sa vie d'adulte. Paul/Michel grandit dans les quartiers suburbains nord, puis se rapproche du Plateau pour rejoindre Ahuntsic, de l'autre côté de l'île.

\section{Document 5}

Déplacements de Paul à Montréal

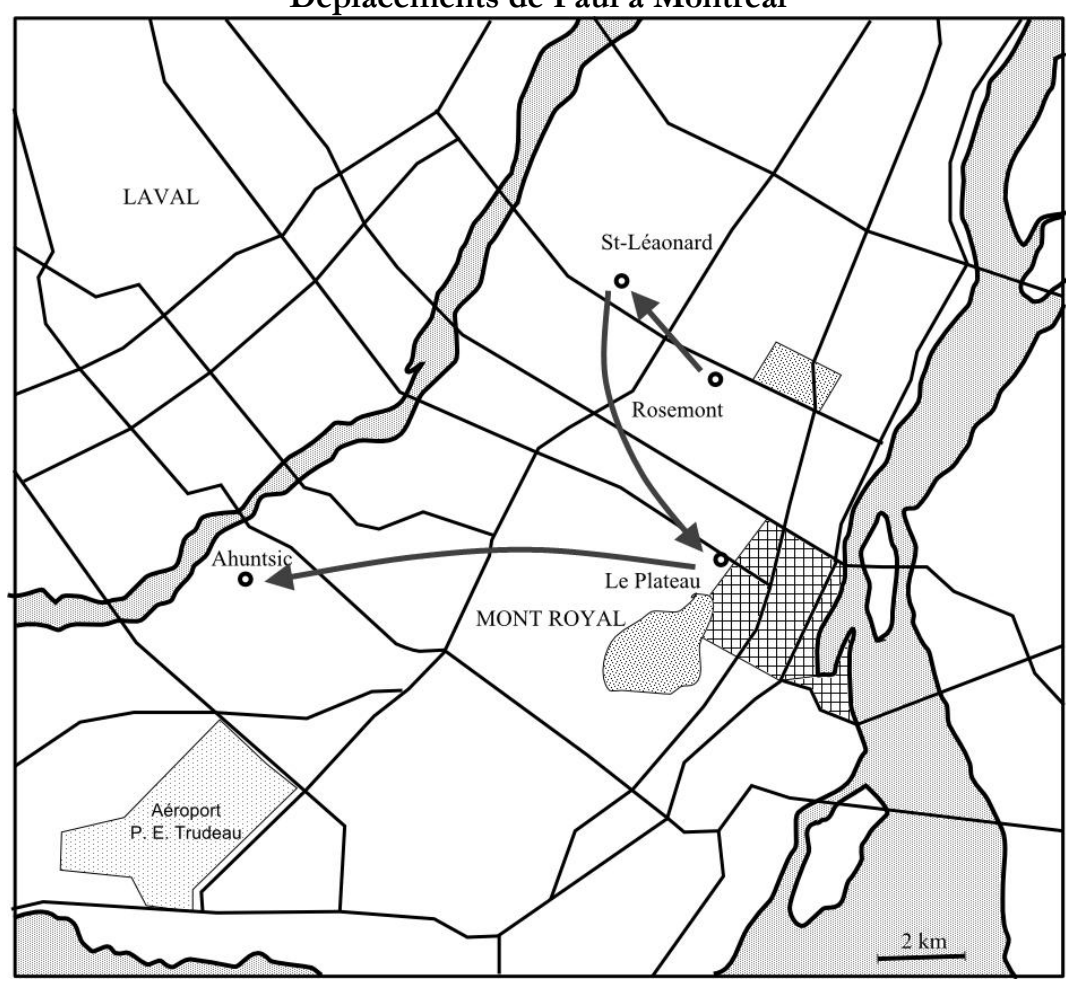

\footnotetext{
${ }^{5}$ GUILBERT, Xavier. »Michel Rabagliati » [en ligne] sur le site $d u 9$, entretien réalisé le 28 janvier 2001 lors du Festival International de la Bande dessinée d'Angoulême [consulté le 16 mars 2011]. Disponible sur le Web. URL $<$ http://www.du9.org/Michel-Rabagliati>

${ }^{6}$ « Le Montréal de Paul » sur le site de L'actualité, août 2010 : http://www2.lactualite.com/multimedia/photoreportage/le-montreal-de-paul/2010-08-02/
} 
La territorialité des quartiers vécus par les personnages se traduit finalement dans les planches par la représentation de lieux, souvent disposés en " réseau » autour du foyer de Paul. Les lieux que dessine Rabagliati et que côtoie Paul sont hors du temps, inscrits dans une réalité, chargés d'affects. Dans Paul en appartement, qui constitue le plus montréalais des albums de la série, de nombreux lieux représentés par l'auteur n'ont d'autre fonction dans le récit sinon de planter ledécor de l'histoire. La page 21, par exemple, est entièrement dédiée à l'évocation de lieux épars sur le Plateau de Montréal. Les monuments funéraires L.Berson \& son (p.21, c.1), en particulier, occupent une place importante puisque la case s'étend sur la bande inférieure. Ce lieu n'a aucun rôle dans le récit, mais il est chargé d'affects et de temporalité pour l'auteur :

Voilà exactement le genre de décor que j’aime dessiner. Une toile de fond qui n'apporte rien à l'histoire proprement dite, mais qui parle de nous et illustre des coins "spéciaux » de notre ville. Tant qu'à faire déambuler des personnages dans la rue, pourquoi ne pas les faire passer devant des endroits historiques ou poétiques ? ${ }^{7}$

\subsection{Paul est québécois}

Les différents épisodes de la série relatent les voyages réalisés par Paul avec ses parents ou avec Lucie autour de Montréal. Ces expéditions ont principalement lieu vers le nord de Montréal dans la région des lacs. Ces espaces montagneux, lacustres et forestiers tranchent avec la vie urbaine des personnages dans l'agglomération montréalaise. Là encore, comme pour Roland Beaulieu, la mobilité est extrême et les grandes distances n'arrêtent pas les personnages. Cette pratique des vastes étendues est bel et bien un véritable témoignage d'une spatialité particulière qui peut être considérée comme propre aux Québécois.

Paul se déplace, franchit des limites topographiques, consomme des espaces, met bout à bout différents lieux qui ont jalonné son enfance. Dans Paul à la campagne (1999), il se rend à 150 $\mathrm{km}$ à l'ouest de Montréal, près de Gatineau, au bord du lac des Chats, dans le chalet des vacances (p.1 à 12). Dans Paul a un travail d'eté (2002), il traverse Montréal (p. 10 à 13), à pied ou en empruntant différents transports en commun ; il rejoint son camp d'été en autocar jusqu'à ValMorin dans l'Ontario, à 300km au nord (p.23 à 27). Dans Paul à la pếche, le couple se rend au bord du lac Taureau pour passer des vacances, à $150 \mathrm{~km}$ de Montréal. Lors de ces déplacements, qui se font souvent sur plusieurs pages, Rabagliati s'évertue à représenter les nombreux paysages traversés, toujours les mêmes et pourtant toujours différents. Ces espaces continus mis bout à bout par divers modes de connexions constituent un véritable réseau qui fait comme une trame.

Document 6 :

Déplacements de Paul autour de Montréal

\footnotetext{
${ }^{7}$ « Le Montréal de Paul » sur le site de L'actualité, août 2010 :

http://www2.lactualite.com/multimedia/photoreportage/le-montreal-de-paul/2010-08-02/8/
} 


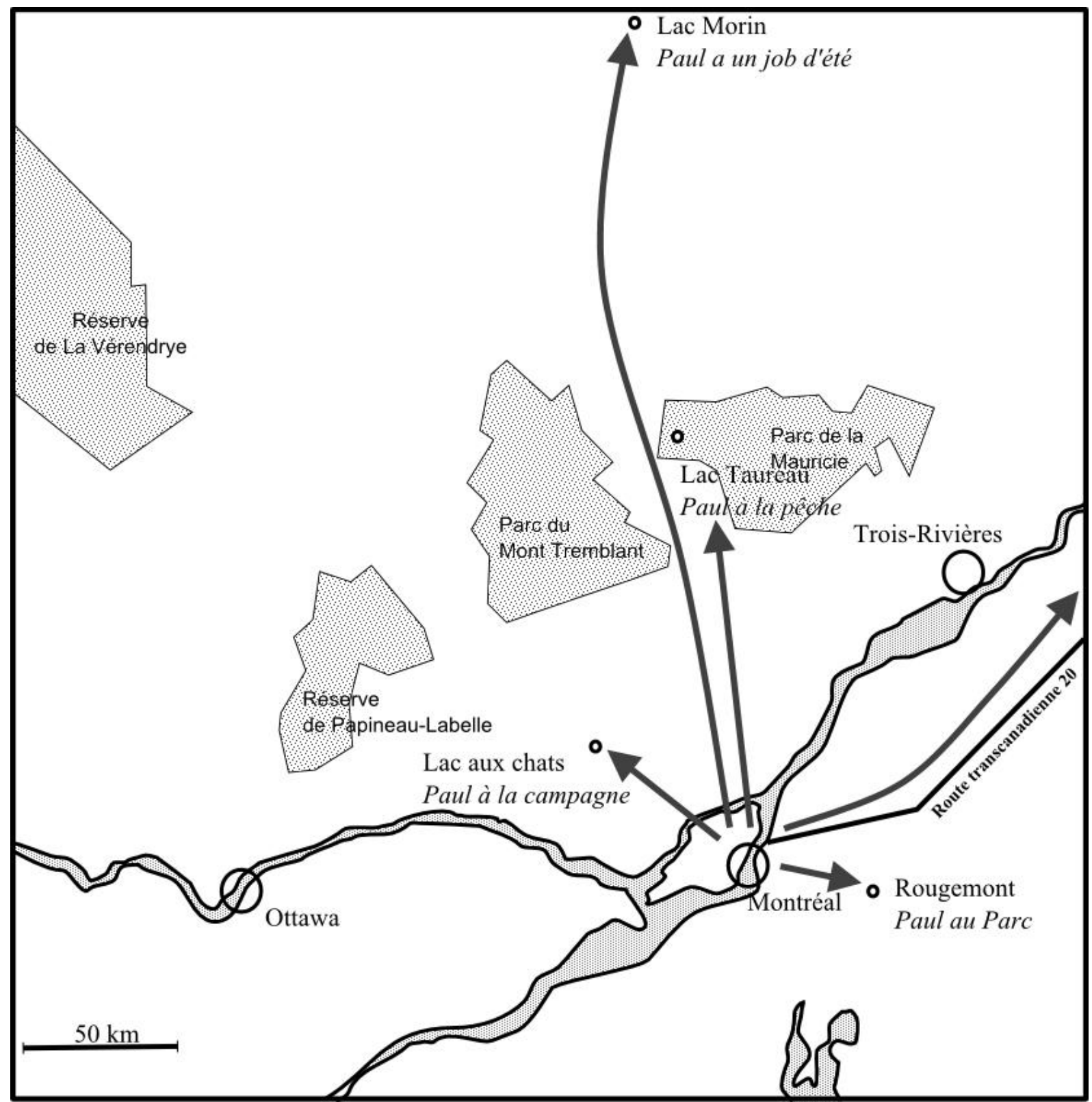

Les ouvrages de la série Paul sont de véritables récits d'espaces. Rabaglati y réinvente le Montréal et le Québec de son enfance, l'intégralité d'un territoire vécu tramé par les itinéraires menant les personnages de lieux en lieux. C'est dans cette spatialité que nous avions qualifiée de topo et réti-graphique dans un récent article consacré au travail de l'auteur québécois ${ }^{8}$, dans la mesure où elle mêle lieux, affectés socialement et sentimentalement, et réseau.

\section{Conclusion}

Pour conclure, nous aimerions terminer par une citation empruntée au géographe Denis Retaillé, invité à réfléchir lors d'un colloque international sur les nouvelles logiques spatiales :

\footnotetext{
Il y a deux manières d'habiter le monde : dans une généalogie ou dans la coprésence. [...] La première manière vise à combler l'abîme de l'existence humaine en expliquant pourquoi je suis là. Elle est guidée par l'esprit historien et tendue entre la Création et le Jugement ; une sorte de millénarisme l'habite. La seconde manière montre plutôt comment je suis là, en se plaçant du
}

8 Sylvie Dardaillon et Christophe Meunier, «La série Paul de Michel Rabagliati : récits d'espaces et de temps », Comicalités [En ligne], Représenter l'auteur de bandes dessinées, mis en ligne le 17 avril 2013, consulté le 02 septembre 2013. URL : http://comicalites.revues.org/1566 ; DOI : 10.4000/comicalites. 1566 
point de vue de l'universel et non pas de l'éternel. Elle est guidée par l'esprit géographique et tendue entre ici et ailleurs, à la recherche de l'unité et de la différence. Autrement dit, l'identité est héritée pour les uns, se construit pour les autres. ${ }^{9}$

Rabagliati a le génie des lieux. Son identité est à la fois un héritage et une construction personnelle. Elle manie les temporalités et les spatialités, l'histoire et la géographie. Il s'agit d'une identité trempée dans une territorialisation forte, une hétérotypie au sens foucaldien. Multiplier les lieux, leur donner une histoire et une importance personnelle, les installer en réseaux concrets et symboliques : voilà les processus identificatoires chez Paul/Rabagliati.

\section{Bibliographie}

1. Corpus

RABAGLIATI, Michel. Paul à la campagne. Montréal : La Pastèque, 1999, 47 p.

RABAGLIATI, Michel. Paul a un travail d'eté. Montréal : La Pastèque, 2002, 151 p.

RABAGLIATI, Michel. Paul en appartement. Montréal : La Pastèque, 2004, 110 p.

RABA GLIATI, Michel. Paul dans le métro. Montréal : La Pastèque, 2005, 91 p.

RABAGLIATI, Michel. Paul à la pếche. Montréal : La Pastèque, 2006, 199 p.

RABA GLIATI, Michel. Paul à Québec. Montréal : La Pastèque, 2009, 187 p.

RABAGLIATI, Michel. Les extras de Paul. Montréal : La Pastèque, 2010, 32 p.

RABAGLIATI, Michel. Paul au parc. Montréal : La Pastèque, 2011, 144 p.

2. Bibliographie critique

AUGÉ, Marc. Non-lieux. Introduction à une anthropologie de la surmodernité. Paris : Seuil, 1992, 150p.

CERTEAU (de), Michel. L'invention du quotidien. Volume 1, Paris : Gallimard, 1990, 350p.

GUILBERT, Xavier. »Michel Rabagliati » [en ligne] sur le site du9, entretien réalisé le 28 janvier 2001 lors du Festival International de la Bande dessinée d'Angoulême [consulté le 16 mars 2011]. Disponible sur le Web. URL

$<$ http://www.du9.org/Michel-Rabagliati>

URBAIN, Jean-Didier. L'idiot du voyage. Histoires de touristes. Paris : Payot, 1993, 349p.

«Le Montréal de Paul » sur le site de L'actualité, août 2010 :

http://www.lactualite.com/culture/le-montreal-de-paul/

\footnotetext{
${ }^{9}$ Denis Retaillé, Penser le monde » dans J. Lévy et M. Lussault (dir.), Logiques de l'espace, esprit des lieux. Géographies à Cerisy, Paris : Belin, 2000, p.283.
} 